\title{
A Two-Parameter Weighted Rama Distribution with Properties and Application
}

\author{
Tasfalem Eyob $^{1}$ and Rama Shanker ${ }^{1^{*}}$ \\ ${ }^{1}$ Department of Statistics, Eritrea Institute of Technology, Asmara, Eritrea \\ * Corresponding author
}

\section{Article Info}

Keywords: Hazard rate function, Goodness of fit, Maximum likelihood estimation, Moments, Mean residual life function, Rama distribution, Stochastic ordering

2010 AMS: 62E05, $62 E 99$

Received: 8 May 2018

Accepted: 29 November 2018

Available online: 28 June 2018

\begin{abstract}
In this paper, a two-parameter weighted Rama distribution which includes one parameter Rama distribution introduced by Shanker [1] has been proposed for modelling real lifetime data. Statistical properties of the distribution including shapes of a probability density function, moments and moment related measures, hazard rate function, mean residual life function, and stochastic orderings have been discussed. The estimation of its parameters has been discussed using the method of maximum likelihood. Application of the proposed distribution has been discussed.
\end{abstract}

\section{Introduction}

The concept of weighted distributions was firstly introduced by Fisher [2] to model ascertainment biases which were later formalized by Rao [3] in a unifying theory for problems where the observations fall in non-experimental, non-replicated and non-random. When an investigator records an observation in nature according to a certain stochastic model, the distribution of the recorded observation will not have the original distribution unless every observation is given an equal chance of being recorded. For example, suppose the original observation $x_{0}$ comes from a distribution having a probability density function (pdf.), $f_{o}\left(x, \theta_{1}\right)$, where $\theta_{1}$ may be a parameter vector and observation $x$ is recorded according to a probability re-weighted by a weight function $w\left(x, \theta_{2}\right)>0, \theta_{2}$ being a new parameter vector, then $x$ comes from a distribution having pdf

$$
f\left(x ; \theta_{1}, \theta_{2}\right)=k w\left(x ; \theta_{2}\right) f_{o}\left(x ; \theta_{2}\right)
$$

where $k$ is a normalizing constant. Recall that such types of distributions are known as weighted distributions. The weighted distributions with a weight function $w\left(x, \theta_{2}\right)=x$ are called length-biased distribution. Patil and Rao [4]-[5] have examined some general probability models leading to weighted probability distributions, discussed their applications and showed the occurrence of $w\left(x, \theta_{2}\right)=x$ in a natural way in problems relating to sampling.

Shanker [1] has introduced Rama distribution for modelling behavioural Science data defined by its pdf and cumulative distribution function (cdf)

$$
\begin{aligned}
& f_{1}(x ; \theta)=\frac{\theta^{4}}{\theta^{3}+6}\left(1+x^{3}\right) e^{-\theta x} ; x>0, \theta>0 \\
& F_{1}(x, \theta)=1-\left[1+\frac{\theta^{3} x^{3}+3 \theta^{2} x^{2}+6 \theta x}{\theta^{3}+6}\right] e^{-\theta x} ; x>0, \theta>0
\end{aligned}
$$

Thus the $r^{\text {th }}$ moment about origin $\mu^{\prime}{ }_{r}$ of Rama distribution (1.1) obtained by Shanker [1] is given by 


$$
\mu_{r}^{\prime}=\frac{r !\left[\theta^{3}+(r+1)(r+2)(r+3)\right]}{\theta^{r}\left(\theta^{3}+6\right)} ; r=1,2,3, \ldots
$$

The first four moments about origin of Rama distribution obtained by Shanker [1] are as follows

$$
\begin{aligned}
& \mu_{1}{ }^{\prime}=\frac{\theta^{3}+24}{\theta\left(\theta^{3}+6\right)} \\
& \mu_{2}{ }^{\prime}=\frac{2\left(\theta^{3}+60\right)}{\theta^{2}\left(\theta^{3}+6\right)} \\
& \mu_{3}{ }^{\prime}=\frac{6\left(\theta^{3}+120\right)}{\theta^{3}\left(\theta^{3}+6\right)} \\
& \mu_{4}{ }^{\prime}=\frac{24\left(\theta^{3}+210\right)}{\theta^{4}\left(\theta^{3}+6\right)} .
\end{aligned}
$$

The moments about mean of Rama distribution are

$$
\begin{aligned}
& \mu_{2}=\frac{\theta^{6}+84 \theta^{3}+144}{\theta^{2}\left(\theta^{3}+6\right)^{2}} \\
& \mu_{3}=\frac{2\left(\theta^{9}+198 \theta^{6}+324 \theta^{3}+864\right)}{\theta^{3}\left(\theta^{3}+6\right)^{3}} \\
& \mu_{4}=\frac{9\left(\theta^{12}+312 \theta^{9}+2304 \theta^{6}+10368 \theta^{3}+10368\right)}{\theta^{4}\left(\theta^{3}+6\right)^{4}} .
\end{aligned}
$$

Shanker [1] has discussed statistical properties including shapes of pdf for varying values of parameter, hazard rate function; mean residual life function, stochastic ordering, mean deviations, order statistic, Bonferroni and Lorenz curves, Renyi entropy measures, and stress-strength reliability of Rama distribution. Shanker [1] has also studied the estimation of the parameter of Rama distribution using both the method of maximum likelihood and the method of the moment along with an application.

In this paper, a two-parameter weighted Rama distribution which includes one parameter Rama distribution proposed by Shanker [1] has been introduced and studied. The statistical properties of the distribution including the coefficient of variation, skewness, kurtosis, index of dispersion, hazard rate function, mean residual life function, and stochastic ordering have been discussed. The method of maximum likelihood has been discussed for estimating parameters. The goodness of fit of the proposed distribution has been discussed with a real lifetime data and it shows a quite satisfactory fit over one parameter life time distributions including exponential, Lindley, Rama and two-parameter lifetime distributions including Gompertz, lognormal, Generalized exponential introduced by Gupta and Kundu [6], weighted Lindley introduced by Ghitany et al [7] and weighted Sujatha distribution introduced by Shanker et al [8].

\section{Weighted Rama Distribution}

The pdf of the weighted Rama distribution (WRD) can be expressed as

$$
f(x ; \theta, \alpha)=K x^{\alpha-1} f_{o}(x ; \theta) ; x>0, \theta>0, \alpha>0
$$

where, $K$ is the normalizing constant and $f_{o}(x ; \theta)$ is the pdf of Rama distribution given in (1.1). Thus the pdf of WRD can be obtained as

$$
f_{2}(x ; \theta, \alpha)=\frac{\theta^{\alpha+3}}{\theta^{3}+\alpha(\alpha+1)(\alpha+2)} \frac{x^{\alpha-1}}{\Gamma(\alpha)}\left(1+x^{3}\right) e^{-\theta x} ; x>0, \theta>0, \alpha>0
$$

where $\theta=$ scale parameter, $\alpha=$ shape parameter and

$$
\Gamma(\alpha)=\int_{0}^{\infty} e^{-y} y^{\alpha-1} d y ; y>0, \alpha>0
$$

is the complete gamma function.

Further, pdf (2.1) can be expressed as a two-component mixture of gamma $(\theta, \alpha)$ and gamma $(\theta, \alpha+3)$ distributions. We have

$$
f_{2}(x ; \theta, \alpha)=p g_{1}(x ; \theta, \alpha)+(1-p) g_{2}(x ; \theta, \alpha+3)
$$


where

$$
\begin{aligned}
& p=\frac{\theta^{3}}{\theta^{3}+\alpha(\alpha+1)(\alpha+2)} \\
& g_{1}(x ; \theta, \alpha)=\frac{\theta^{\alpha}}{\Gamma(\alpha)} e^{-\theta x} x^{\alpha-1} \\
& g_{2}(x ; \theta, \alpha+3)=\frac{\theta^{\alpha+3}}{\Gamma(\alpha+3)} e^{-\theta x} x^{(\alpha+3)-1}
\end{aligned}
$$

The behaviour of the pdf of WRD for varying values of parameters $\theta$ and $\alpha$ are shown in Figure 2.1.
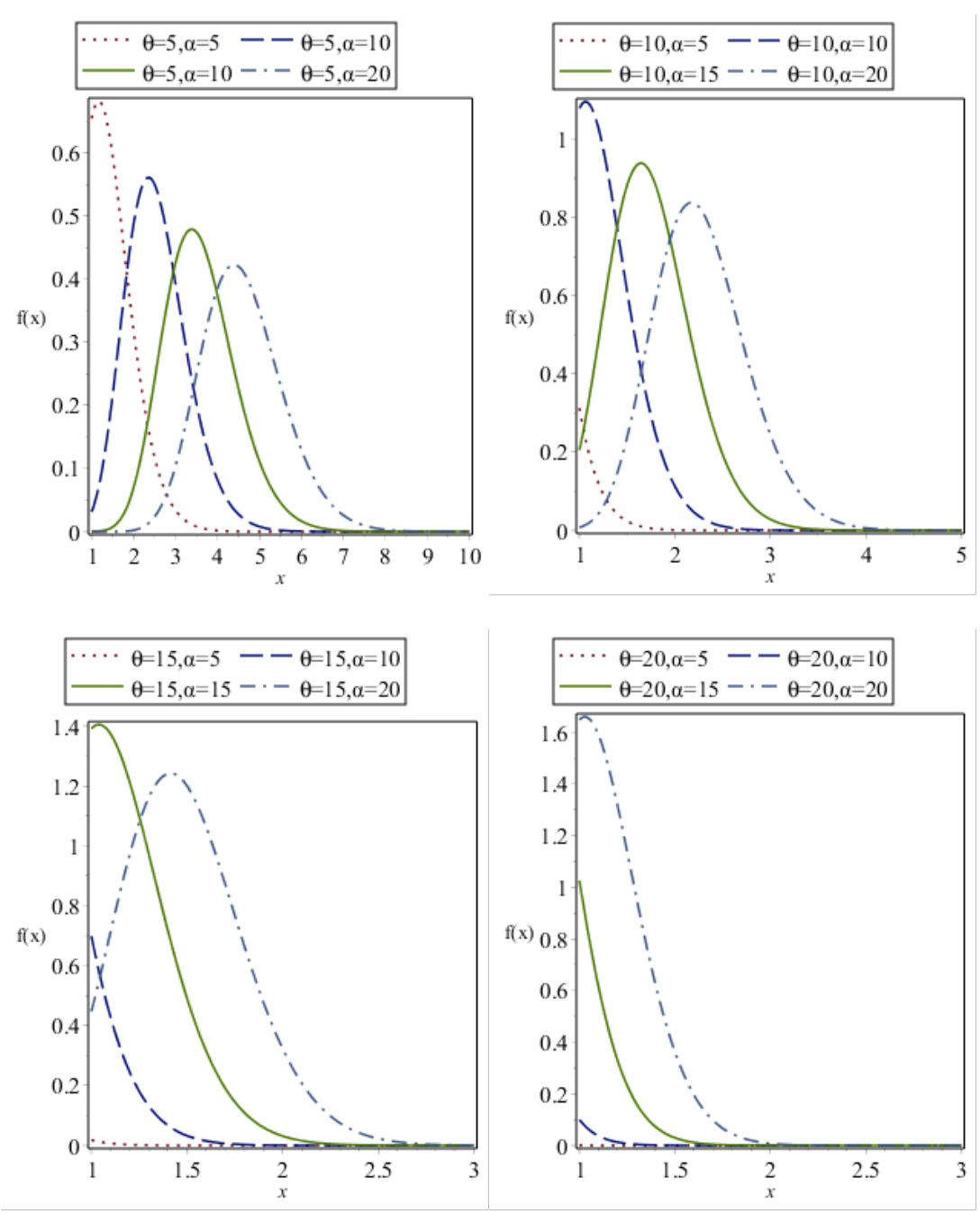

Figure 2.1: Behavior of the pdf of WRD for various values of the parameters $\theta$ and $\alpha$

The cdf of WRD can be obtained as

$$
F_{2}(x ; \theta, \alpha)=1-\left[\begin{array}{c}
(\theta x)^{\alpha}\left\{(\theta x)^{2}+(\theta x)(\alpha+2)+(\alpha+1)(\alpha+2)\right\} e^{-\theta x} \\
\frac{+\left\{\theta^{3}+\alpha(\alpha+1)(\alpha+2)\right\} \Gamma(\alpha, \theta x)}{\left[\theta^{3}+\alpha(\alpha+1)(\alpha+2)\right] \Gamma(\alpha)}
\end{array}\right] ; x>0, \theta>0, \alpha>0
$$

where $\Gamma(\alpha, \theta x)$ is the upper incomplete gamma function defined by

$$
\Gamma(\alpha, z)=\int_{z}^{\infty} e^{-y} y^{\alpha-1} d y ; \quad y \geq 0, \quad \alpha>0 .
$$

The behaviour of the cdf of WRD for varying values of the parameters $\theta$ and $\alpha$ are shown in Figure 2.2. 

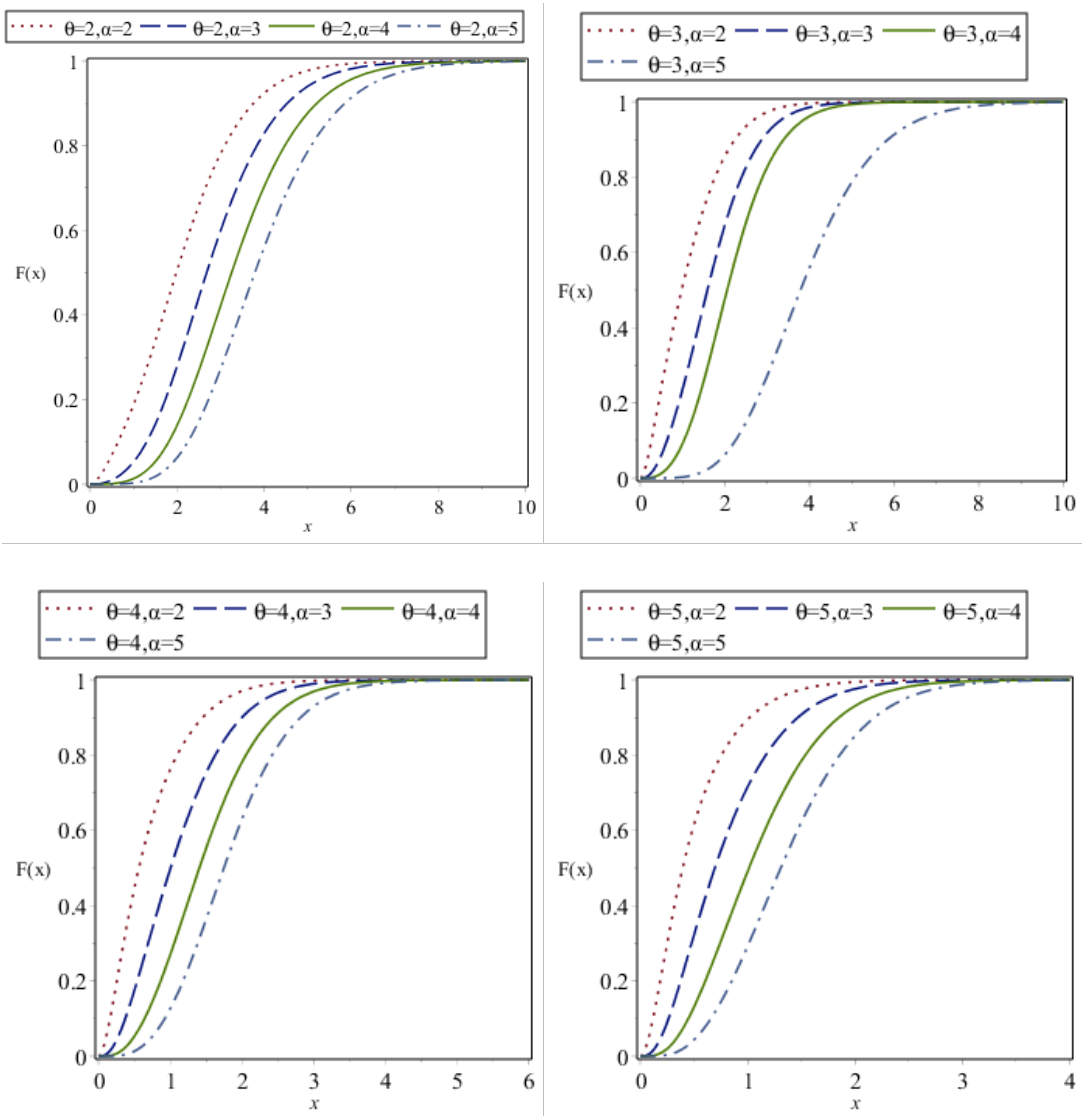

Figure 2.2: Behavior of the cdf of WRD for various values of the parameters $\theta$ and $\alpha$

\section{Statistical Constants}

The $r^{\text {th }}$ moment about origin, $\mu^{\prime}{ }_{r}$ of WRD (2.1) can be obtained as

$$
\begin{aligned}
& \mu_{r}^{\prime}=E\left(X^{r}\right)=\int_{0}^{\infty} x^{r} f_{2}(x ; \theta, \alpha) d x=\int_{0}^{\infty} x^{r} \frac{\theta^{\alpha+3} x^{\alpha-1}\left(1+x^{3}\right)}{\Gamma(\alpha)\left[\theta^{3}+\alpha(\alpha+1)(\alpha+2)\right]} e^{-\theta x} d x \\
& =\frac{\Gamma(\alpha+r)}{\Gamma(\alpha)} \frac{\theta^{3}+(\alpha+r)(\alpha+r+1)(\alpha+r+2)}{\theta^{r}\left[\theta^{3}+\alpha(\alpha+1)(\alpha+2)\right]} ; r=1,2,3, \ldots
\end{aligned}
$$

The first four moments about origin of WRD, after substituting $r=1,2,3$ and 4 in (3.1) are obtained as

$$
\begin{aligned}
& \mu_{1}^{\prime}=\frac{\alpha\left\{\theta^{3}+(\alpha+1)(\alpha+2)(\alpha+3)\right\}}{\theta\left[\theta^{3}+\alpha(\alpha+1)(\alpha+2)\right]} \\
& \mu_{2}^{\prime}=\frac{\alpha(\alpha+1)\left\{\theta^{3}+(\alpha+2)(\alpha+3)(\alpha+4)\right\}}{\theta^{2}\left[\theta^{3}+\alpha(\alpha+1)(\alpha+2)\right]} \\
& \mu_{3}^{\prime}=\frac{\alpha(\alpha+1)(\alpha+2)\left\{\theta^{3}+(\alpha+3)(\alpha+4)(\alpha+5)\right\}}{\theta^{3}\left[\theta^{3}+\alpha(\alpha+1)(\alpha+2)\right]} \\
& \mu_{4}^{\prime}=\frac{\alpha(\alpha+1)(\alpha+2)(\alpha+3)\left\{\theta^{3}+(\alpha+4)(\alpha+5)(\alpha+6)\right\}}{\theta^{4}\left[\theta^{3}+\alpha(\alpha+1)(\alpha+2)\right]} .
\end{aligned}
$$

Now using the relationship $\mu_{r}=E\left(X-\mu_{1}^{\prime}\right)^{r}=\sum_{k=0}^{r}\left(\begin{array}{c}r \\ k\end{array}\right) \mu_{k}^{\prime}\left(-\mu_{1}^{\prime}\right)^{r-k}$ between moments about mean and moments about origin, the moments about the mean of WRD are obtained as 


$$
\mu_{2}=\frac{\alpha\left\{\theta^{6}+2\left(\alpha^{3}+9 \alpha^{2}+20 \alpha+12\right) \theta^{3}+\left(\alpha^{6}+9 \alpha^{5}+31 \alpha^{4}+51 \alpha^{3}+40 \alpha^{2}+12 \alpha\right)\right\}}{\theta^{2}\left(\theta^{3}+\alpha^{3}+3 \alpha^{2}+2 \alpha\right)^{2}}
$$

$\mu_{3}=\frac{2 \alpha\left\{\begin{array}{l}\theta^{9}+3\left(\alpha^{3}+13 \alpha^{2}+32 \alpha+20\right) \theta^{6}+3\left(\alpha^{6}+8 \alpha^{5}+25 \alpha^{4}+38 \alpha^{3}+28 \alpha^{2}+8 \alpha\right) \theta^{3} \\ +\left(\alpha^{9}+12 \alpha^{8}+60 \alpha^{7}+162 \alpha^{6}+255 \alpha^{5}+234 \alpha^{4}+116 \alpha^{3}+24 \alpha^{2}\right)\end{array}\right\}}{\theta^{3}\left(\theta^{3}+\alpha^{3}+3 \alpha^{2}+2 \alpha\right)^{3}}$

$\mu_{4}=\frac{3 \alpha\left\{\begin{array}{l}(\alpha+2) \theta^{12}+4\left(\alpha^{4}+11 \alpha^{3}+56 \alpha^{2}+106 \alpha+60\right) \theta^{9} \\ +3\left(2 \alpha^{6}+34 \alpha^{5}+198 \alpha^{4}+550 \alpha^{3}+792 \alpha^{2}+568 \alpha+160\right) \alpha \theta^{6} \\ +4\left(\alpha^{8}+20 \alpha^{7}+156 \alpha^{6}+642 \alpha^{5}+1551 \alpha^{4}+2274 \alpha^{3}+1988 \alpha^{2}+952 \alpha+198\right) \alpha^{2} \theta^{3} \\ +\left(\begin{array}{c}\alpha^{13}+20 \alpha^{12}+173 \alpha^{11}+856 \alpha^{10}+2691 \alpha^{9}+5628 \alpha^{8} \\ +7943 \alpha^{7}+7480 \alpha^{6}+4504 \alpha^{5}+1568 \alpha^{4}+240 \alpha^{3}\end{array}\right)\end{array}\right.}{\theta^{4}\left(\theta^{3}+\alpha^{3}+3 \alpha^{2}+2 \alpha\right)^{4}}$.

It can be easily shown that at $\alpha=1$, the moments about the origin and the moments about mean of WRD reduces to the corresponding moments of Rama distribution.

The expressions for coefficient variation (C.V.) coefficient of skewness $\left(\sqrt{\beta_{1}}\right)$, the coefficient of kurtosis $\left(\beta_{2}\right)$ and index of dispersion $(\gamma)$ of WRD is thus given as

$$
C . V=\frac{\sigma}{\mu_{1}{ }^{\prime}}=\frac{\sqrt{\alpha\left\{\theta^{6}+\left(2 \alpha^{3}+18 \alpha^{2}+40 \alpha+24\right) \theta^{3}+\alpha^{6}+9 \alpha^{5}+31 \alpha^{4}+51 \alpha^{3}+40 \alpha^{2}+12 \alpha\right\}}}{\alpha\left(\theta^{3}+\alpha^{3}+6 \alpha^{2}+11 \alpha+6\right)}
$$

$$
\sqrt{\beta_{1}}=\frac{\mu_{3}}{\mu_{2}^{3 / 2}}=\frac{2 \alpha\left\{\begin{array}{l}
\theta^{9}+\left(3 \alpha^{3}+39 \alpha^{2}+96 \alpha+60\right) \theta^{6}+\left(3 \alpha^{6}+24 \alpha^{5}+75 \alpha^{4}+114 \alpha^{3}+84 \alpha^{2}+24 \alpha\right) \theta^{3} \\
\alpha^{9}+12 \alpha^{8}+60 \alpha^{7}+162 \alpha^{6}+255 \alpha^{5}+234 \alpha^{4}+116 \alpha^{3}+24 \alpha^{2}
\end{array}\right\}}{\left\{\alpha\left(\theta^{6}+\left(2 \alpha^{3}+18 \alpha^{2}+40 \alpha+24\right) \theta^{3}+\alpha^{6}+9 \alpha^{5}+31 \alpha^{4}+51 \alpha^{3}+40 \alpha^{2}+12 \alpha\right)\right\}^{3 / 2}}
$$$$
\beta_{2}=\frac{\mu_{4}}{\mu_{2}^{2}}=\frac{3(\alpha+2)\left\{\begin{array}{l}
\theta^{12}+\left(4 \alpha^{3}+36 \alpha^{2}+152 \alpha+120\right) \theta^{9}+\left(6 \alpha^{6}+90 \alpha^{5}+414 \alpha^{4}+822 \alpha^{3}+732 \alpha^{2}+240 \alpha\right) \theta^{6} \\
+\left(4 \alpha^{9}+72 \alpha^{8}+480 \alpha^{7}+1608 \alpha^{6}+2988 \alpha^{5}+3120 \alpha^{4}+1712 \alpha^{3}+384 \alpha^{2}\right) \theta^{3} \\
+\left(\alpha^{12}+18 \alpha^{11}+137 \alpha^{10}+582 \alpha^{9}+1527 \alpha^{8}+2574 \alpha^{7}+2795 \alpha^{6}+1890 \alpha^{5}+724 \alpha^{4}+120 \alpha^{3}\right)
\end{array}\right\}}{\left\{\alpha\left(\theta^{6}+\left(2 \alpha^{3}+18 \alpha^{2}+40 \alpha+24\right) \theta^{3}+\alpha^{6}+9 \alpha^{5}+31 \alpha^{4}+51 \alpha^{3}+40 \alpha^{2}+12 \alpha\right)^{2}\right\}}
$$

$$
\gamma=\frac{\sigma^{2}}{\mu_{1}^{\prime}}=\frac{\left\{\theta^{6}+\left(2 \alpha^{3}+18 \alpha^{2}+40 \alpha+24\right) \theta^{3}+\alpha^{6}+9 \alpha^{5}+31 \alpha^{4}+51 \alpha^{3}+40 \alpha^{2}+12 \alpha\right\}}{\theta\left(\theta^{3}+\alpha^{3}+3 \alpha^{2}+2 \alpha\right)\left(\theta^{3}+\alpha^{3}+6 \alpha^{2}+11 \alpha+6\right)} .
$$

The behaviour of the coefficient of variation (C.V.), the coefficient of skewness (C.S.), the coefficient of kurtosis (C.K.) and index of dispersion (I.D.) of WRD have been prepared for varying values of the parameters $\theta$ and $\alpha$ and presented in Figure 3.1. 

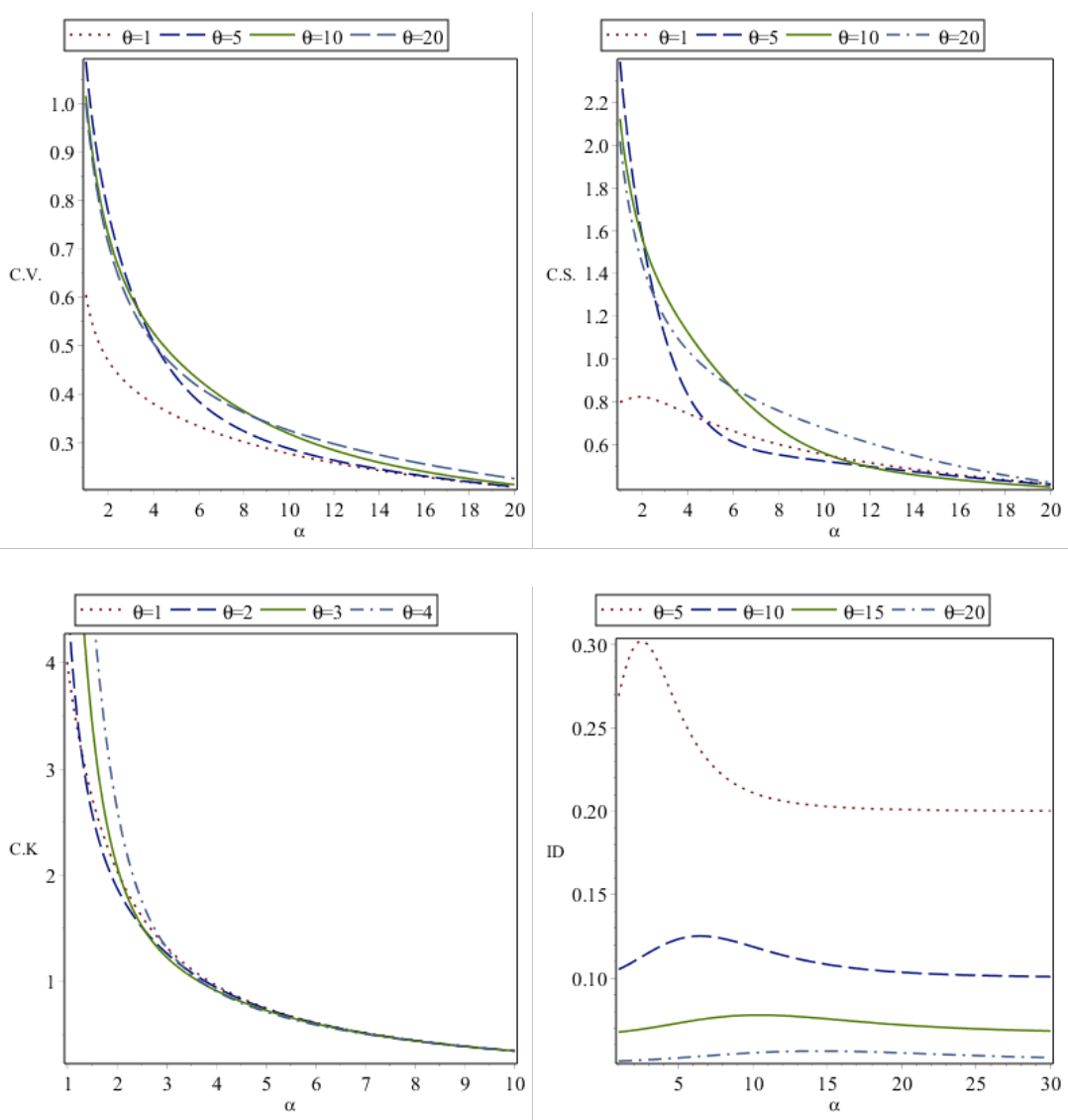

Figure 3.1: Behavior of C.V., C.S., C.K., and I.D of WRD for varying values of parameters $\theta$ and $\alpha$

\section{Survival Function ans Hazard Rate Function}

The survival (reliability) function of WRD can be obtained as

$$
S(x ; \theta, \alpha)=1-F_{2}(x ; \theta, \alpha)=\frac{\left\{\begin{array}{c}
(\theta x)^{\alpha}\left[(\theta x)^{2}+(\alpha+2)(\theta x)+(\alpha+1)(\alpha+2)\right] e^{-\theta x} \\
+\left[\theta^{3}+\alpha(\alpha+1)(\alpha+2)\right] \Gamma(\alpha, \theta x)
\end{array}\right\}}{\left[\theta^{3}+\alpha(\alpha+1)(\alpha+2)\right] \Gamma(\alpha)} .
$$

The hazard (or failure) rate function, $h(x)$ of WRD is thus obtained as

$$
h(x)=\frac{f(x ; \theta, \alpha)}{S(x ; \theta, \alpha)}=\frac{\theta^{\alpha+3} x^{\alpha-1}\left(1+x^{3}\right) e^{-\theta x}}{\left\{\begin{array}{l}
{\left[\theta^{3}+\alpha(\alpha+1)(\alpha+2)\right] \Gamma(\alpha, \theta x)} \\
+(\theta x)^{\alpha}\left[(\theta x)^{2}+(\theta x)(\alpha+2)+(\alpha+1)(\alpha+2)\right] e^{-\theta x}
\end{array}\right\}} ; x>0, \theta>0, \alpha>0 .
$$



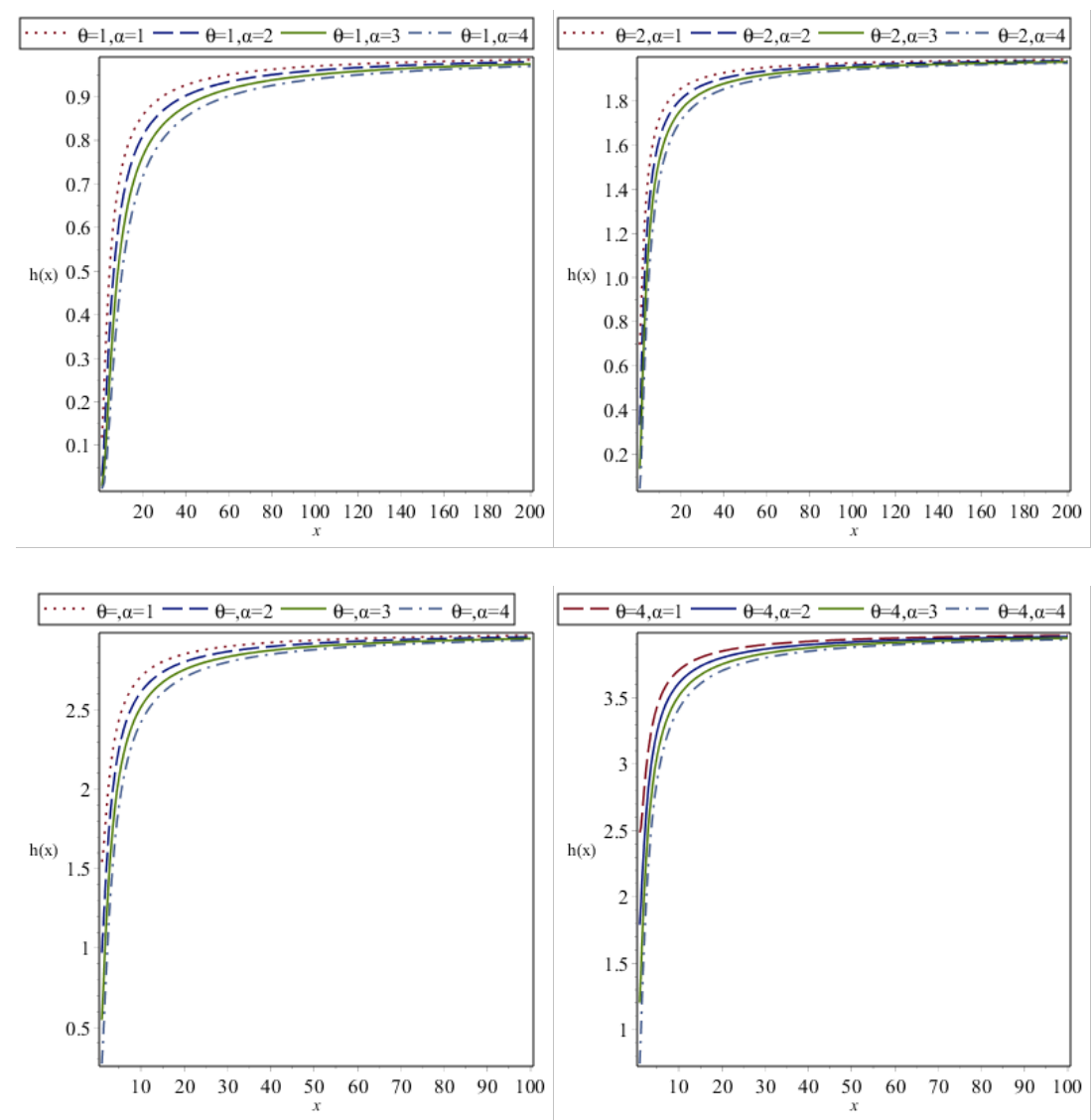

Figure 4.1: Behavior of C.V., C.S., C.K., and I.D of WRD for varying values of parameters $\theta$ and $\alpha$

\section{Mean Residual Life Function}

The mean residual life function $\mu(x)=E(X-x \mid X>x)$ of the WRD can be obtained as

$$
\begin{aligned}
\mu(x)= & \frac{1}{S(x ; \theta, \alpha)} \int_{x}^{\infty} y f_{2}(y ; \theta, \alpha) d y-x \\
& =\frac{\left\{\begin{array}{l}
(\theta x)^{\alpha}\left\{(\theta x)^{2}+2(\alpha+2)(\theta x)+\theta^{3}+(\alpha+1)(\alpha+2)(\alpha+3)\right\} e^{\theta x} \\
+\left[\left\{\alpha \theta^{3}+\alpha(\alpha+1)(\alpha+2)(\alpha+3)\right\}-\theta x\left\{\theta^{3}+\alpha(\alpha+1)(\alpha+2)\right\}\right] \Gamma(\alpha, \theta x)
\end{array}\right\}}{\theta\left[(\theta x)^{\alpha}\left\{(\theta x)^{2}+(\alpha+2)(\theta x)+(\alpha+1)(\alpha+2)\right\} e^{-\theta x}+\left\{\theta^{3}+\alpha(\alpha+1)(\alpha+2)\right\} \Gamma(\alpha, \theta x)\right]} .
\end{aligned}
$$

It can be easily shown that

$$
\mu(0)=\frac{\alpha\left[\theta^{3}+(\alpha+1)(\alpha+2)(\alpha+3)\right]}{\theta\left[\theta^{3}+\alpha(\alpha+1)(\alpha+2)\right]}=\mu_{1}^{\prime}
$$



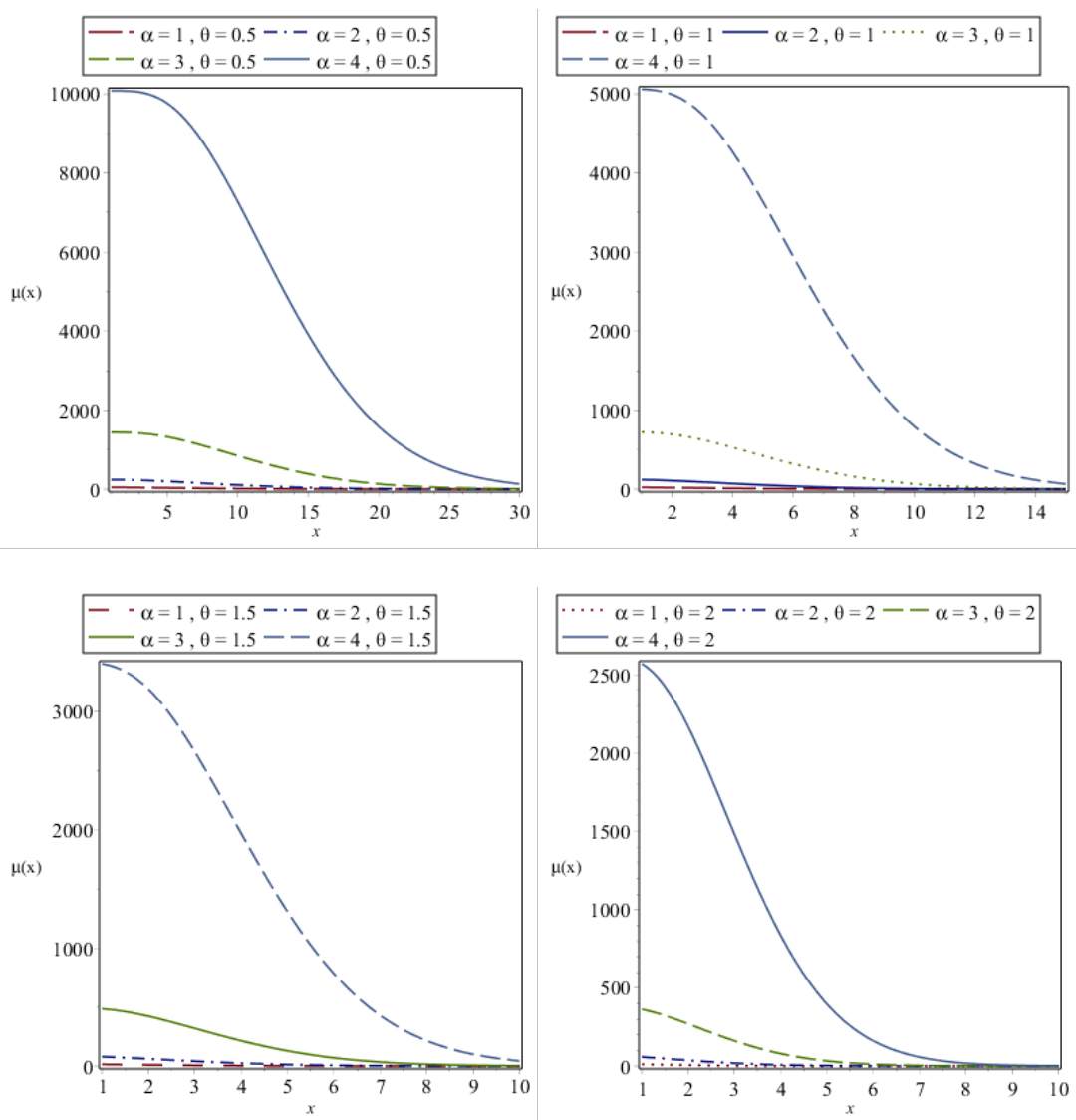

Figure 5.1: Behavior of $\mu(x)$ of the WRD for varying values of parameters $\theta$ and $\alpha$

\section{Stochastic Ordering}

The stochastic ordering of positive continuous random variables is an important tool for examining their comparative behaviour. A random variable $X$ is said to be smaller than a random variable $Y$ in the

- stochastic order $\left(X \leq_{s t} Y\right)$ if $F_{X}(x) \geq F_{Y}(x)$ for all $\mathrm{x}$

- hazard rate order $\left(X \leq_{h r} Y\right)$ if $h_{X}(x) \geq h_{Y}(x)$ for all $\mathrm{x}$

- mean residual life order $\left(X \leq_{m r l} Y\right)$ if $m_{X}(x) \leq m_{Y}(x)$ for all $\mathrm{x}$

- likelihood ratio order $\left(X \leq_{l r} Y\right)$ if $\frac{f_{X}(x)}{f_{Y}(x)}$ decreases in $\mathrm{x}$.

The following important interpretations due to Shaked and Shanthikumar [10] are well known for establishing the stochastic ordering of distributions.

$$
\begin{aligned}
& X \leq_{l r} Y \Rightarrow X \leq_{h r} Y \Rightarrow X \leq_{m r l} Y \\
& \Downarrow \\
& X \leq_{s t} Y \text {. }
\end{aligned}
$$

The WRD is ordered with respect to the strongest 'likelihood ratio' ordering as shown in the following theorem.

Theorem 6.1. Let $X^{\sim} W R D\left(\theta_{1}, \alpha_{1}\right)$ and $Y^{\sim} W R D\left(\theta_{2}, \alpha_{2}\right)$. If $\theta_{1}>\theta_{2}$ and $\alpha_{1}=\alpha_{2}\left(\right.$ or $\alpha_{1}<\alpha_{2}$ and $\left.\theta_{1}=\theta_{2}\right)$, then $X \leq{ }_{l r} Y$ and hence $X \leq{ }_{h r} Y$, $X \leq_{m r l} Y$ and $X \leq_{s t} Y$.

Proof. We have

$$
\frac{f_{X}\left(x ; \theta_{1}, \alpha_{1}\right)}{f_{Y}\left(x ; \theta_{2}, \alpha_{2}\right)}=\frac{\theta_{1}^{\alpha_{1}+3}\left(\theta_{2}^{3}+\alpha_{2}^{3}+3 \alpha_{2}^{2}+2 \alpha_{2}\right) \Gamma\left(\alpha_{2}\right)}{\theta_{2}^{\alpha_{2}+3}\left(\theta_{1}^{3}+\alpha_{1}^{3}+3 \alpha_{1}^{2}+2 \alpha_{1}\right) \Gamma\left(\alpha_{1}\right)} x^{\alpha_{1}-\alpha_{2}} e^{-\left(\theta_{1}-\theta_{2}\right) x} .
$$

Now,

$$
\ln \frac{f_{X}\left(x ; \theta_{1}, \alpha_{1}\right)}{f_{Y}\left(x ; \theta_{2,} \alpha_{2}\right)}=\ln \left(\frac{\theta_{1}^{\alpha_{1}+3}\left(\theta_{2}^{3}+\alpha_{2}^{3}+3 \alpha_{2}^{2}+2 \alpha_{2}\right) \Gamma\left(\alpha_{2}\right)}{\theta_{2}^{\alpha_{2}+3}\left(\theta_{1}^{3}+\alpha_{1}^{3}+3 \alpha_{1}^{2}+2 \alpha_{1}\right) \Gamma\left(\alpha_{1}\right)}\right)+\left(\alpha_{1}-\alpha_{2}\right) \ln x-\left(\theta_{1}-\theta_{2}\right) x .
$$

This gives

$$
\frac{d}{d x} \ln \left(\frac{f_{X}\left(x ; \theta_{1}, \alpha_{1}\right)}{f_{Y}\left(x ; \theta_{2}, \alpha_{2}\right)}\right)=\frac{\alpha_{1}-\alpha_{2}}{x}-\left(\theta_{1}-\theta_{2}\right) .
$$


Thus, if $\left(\alpha_{1}=\alpha_{2}\right.$ and $\left.\theta_{1} \geq \theta_{2}\right)$ or $\left(\alpha_{1}<\alpha_{2}\right.$ and $\left.\theta_{1} \geq \theta_{2}\right)$,then $\frac{d}{d x} \ln \left(\frac{f_{X}\left(x ; \theta_{1}, \alpha_{1}\right)}{f_{Y}\left(x ; \theta_{2}, \alpha_{2}\right)}\right)<0$. This means that $X \leq_{l r} Y$ and hence $X \leq{ }_{h r} Y, X \leq{ }_{m r l} Y$ and $X \leq_{s t} Y$. This shows flexibility of WRD over Rama distribution.

\section{Maximum Likelihood Estimation of Parameters}

Let $\left(x_{1}, x_{2}, x_{3}, \ldots, x_{n}\right)$ be a random sample of size $n$ from WRD (2.1). The likelihood function, $\mathrm{L}$ of WRD is given by

$$
L=\left[\frac{\theta^{\alpha+3}}{\Gamma(\alpha)\left\{\theta^{3}+\alpha^{3}+3 \alpha^{2}+2 \alpha\right\}}\right]^{n} \prod_{i=1}^{n} x_{i}^{\alpha-1}\left(1+x_{i}^{3}\right) e^{-n \theta \bar{x}}
$$

The natural log likelihood function is thus obtained as

$$
\ln L=n\left[(\alpha+3) \ln \theta-\ln \left\{\theta^{3}+\alpha(\alpha+1)(\alpha+2)\right\}-\ln \Gamma(\alpha)\right]+(\alpha-1) \sum_{i=1}^{n} \ln \left(x_{i}\right)+\sum_{i=1}^{n} \ln \left(1+x_{i}^{3}\right)-n \theta \bar{x}
$$

The maximum likelihood estimates $(\widehat{\theta}$,$) of (\theta, \alpha)$ is the solution of the following log likelihood equations.

$$
\begin{aligned}
& \frac{\partial \ln L}{\partial \theta}=\frac{n(\alpha+3)}{\theta}-\frac{3 n \theta^{2}}{\theta^{3}+\alpha^{3}+3 \alpha^{2}+2 \alpha}-n \bar{x}=0 \\
& \frac{\partial \ln L}{\partial \alpha}=n \ln \theta-\frac{n\left(3 \alpha^{2}+6 \alpha+2\right)}{\theta^{3}+\alpha^{3}+3 \alpha^{2}+2 \alpha}-n \psi(\alpha)+\sum_{i=1}^{n} \ln x_{i}=0
\end{aligned}
$$

where $\bar{x}$ is the sample mean and $\psi(\alpha)$ is the digamma function defined as

$$
\psi(\alpha)=\frac{d}{d \alpha} \ln \Gamma(\alpha)
$$

The MLE's $(\hat{\theta}, \hat{\alpha})$ of parameters of WRD $(\theta, \alpha)$ can be computed directly by solving the natural log likelihood equation using NewtonRaphson iteration available in R-software till sufficiently close estimates of $\hat{\theta}$ and $\hat{\alpha}$ are obtained. In this paper, initial values of $\theta$ and $\alpha$ are taken $\theta=0.5$ and $\alpha=1.5$, respectively.

\section{A Numerical Example}

A numerical example of real lifetime data has been presented to test the goodness of fit of WRD over other one parameter and two parameter life time distribution. The following data represent the tensile strength, measured in GPa, of 69 carbon fibers tested under tension at gauge lengths of 20mm, available in Bader and Priest [9].

$\begin{array}{llllllllllll}1.312 & 1.314 & 1.479 & 1.552 & 1.700 & 1.803 & 1.861 & 1.865 & 1.944 & 1.958 & 1.966 & 1.997 \\ 2.006 & 2.021 & 2.027 & 2.055 & 2.063 & 2.098 & 2.140 & 2.179 & 2.224 & 2.240 & 2.253 & 2.270 \\ 2.272 & 2.274 & 2.301 & 2.301 & 2.359 & 2.382 & 2.382 & 2.426 & 2.434 & 2.435 & 2.478 & 2.490 \\ 2.511 & 2.514 & 2.535 & 2.554 & 2.566 & 2.570 & 2.586 & 2.629 & 2.633 & 2.642 & 2.648 & 2.684 \\ 2.697 & 2.726 & 2.770 & 2.773 & 2.800 & 2.809 & 2.818 & 2.821 & 2.848 & 2.880 & 2.954 & 3.012 \\ 3.067 & 3.084 & 3.090 & 3.096 & 3.128 & 3.233 & 3.433 & 3.585 & 3.585 & & & \end{array}$

For this data set, WRD has been fitted along with one parameter exponential, Lindley, Rama distributions and two - parameter gamma distribution Gompertz distribution, generalized exponential distribution (GED) introduced by Gupta and Kundu [6], lognormal distribution ,weighted Sujatha distribution (WSD) introduced by Shanker and Shukla [8] and WLD. The pdf and cdf of gamma, Gompertz, lognormal, GED, WSD and WLD are presented in table 1. The ML estimates, values of $-2 \ln L$, Akaike Information criteria (AIC), K-S statistics and p-value of the fitted distributions are presented in table 2. The AIC and K-S Statistics are computed using the following formulae: $A I C=-2 \ln L+2 k$ and K-S $=\operatorname{Sup}_{x}\left|F_{n}(x)-F_{0}(x)\right|$, where $k=$ the number of parameters, $n=$ the sample size, $F_{n}(x)$ is the empirical (sample) cumulative distribution function, and $F_{0}(x)$ is the theoretical cumulative distribution function. The best distribution is the distribution corresponding to lower values of $-2 \ln L$, AIC, and K-S statistics. 


\begin{tabular}{|c|c|c|}
\hline Distributions & pdf & cdf \\
\hline WSD & $f(x ; \theta, \alpha)=\frac{\theta^{\alpha+2}}{\theta^{2}+\alpha \theta+\alpha(\alpha+1)} \frac{x^{\alpha-1}}{\Gamma(\alpha)}\left(1+x+x^{2}\right) e^{-\theta x}$ & $F(x ; \theta, \alpha, \beta)=1-\frac{\left\{\theta^{2}+\alpha \theta+\alpha(\alpha+1)\right\} \Gamma(\alpha, \theta x)+(\theta x)^{\alpha}(\theta x+\theta+\alpha+1) e^{-\theta x}}{\left\{\theta^{2}+\alpha \theta+\alpha(\alpha+1)\right\} \Gamma(\alpha)}$ \\
\hline WLD & $f(x ; \theta, \alpha)=\frac{\theta^{\alpha+1}}{(\theta+\alpha)} \frac{x^{\alpha-1}}{\Gamma(\alpha)}(1+x) e^{-\theta x}$ & $F(x ; \theta, \alpha)=1-\frac{(\theta+\alpha) \Gamma(\alpha, \theta x)+(\theta x)^{\alpha} e^{-\theta x}}{(\theta+\alpha) \Gamma(\alpha)}$ \\
\hline GED & $f(x ; \theta, \alpha)=\theta \alpha\left(1-e^{-\theta x}\right)^{\alpha-1} e^{-\theta x}$ & $F(x ; \theta, \alpha)=\left(1-e^{-\theta x}\right)^{\alpha}$ \\
\hline Gamma & $f(x ; \theta, \alpha)=\frac{\theta^{\alpha}}{\Gamma(\alpha)} e^{-\theta x} x^{\alpha-1}$ & $F(x ; \theta, \alpha)=\phi\left(\frac{\log x-\theta}{\Gamma}\right)$ \\
\hline Lognormal & $f(x ; \theta, \alpha)=\frac{1}{\sqrt{2 \pi} \alpha x} e^{-\frac{1}{2}\left(\frac{\log x-\theta}{\alpha}\right)^{2}}$ & $F(x ; \theta)=1-\left[1+\frac{\theta x}{\theta+1}\right] e^{-\theta x}$ \\
\hline Lindley & $f(x ; \theta)=\frac{\theta^{2}}{\theta+1}(1+x) e^{-\theta x}$ & $F(x ; \theta, \alpha)=1-e^{-\frac{\theta}{\alpha}\left(e^{\alpha x}-1\right)}$ \\
\hline Gompertz & $f(x ; \theta, \alpha)=\theta e^{\alpha x-\frac{\theta}{\alpha}\left(e^{\alpha x}-1\right)}$ & $F$ \\
\hline
\end{tabular}

Table 1: The pdf and the cdf of fitted distributions

\begin{tabular}{|c|c|c|c|c|c|c|}
\hline \multirow{2}{*}{ Distribution } & \multicolumn{2}{|c|}{ Team sheet } & \multirow{2}{*}{$-2 \ln L$} & AIC & K-S & P-value \\
\cline { 2 - 4 } & $\hat{\theta}$ & $\hat{\alpha}$ & & & & \\
\hline WRD & 9.5764 & 20.7494 & 98.76 & 102.76 & 0.055 & 0.983 \\
\hline WSD & 9.7387 & 22.3612 & 99.99 & 103.99 & 0.057 & 0.975 \\
\hline WLD & 9.6265 & 22.8938 & 101.95 & 105.95 & 0.059 & 0.973 \\
\hline Gamma & 9.5380 & 23.3820 & 100.07 & 104.07 & 0.058 & 0.962 \\
\hline GED & 2.0331 & 87.2847 & 109.24 & 113.24 & 0.087 & 0.613 \\
\hline Lognormal & 0.8751 & 0.2124 & 102.72 & 106.73 & 0.103 & 0.713 \\
\hline Gompertz & 0.0080 & 2.0420 & 107.25 & 111.250 & 0.085 & 0.673 \\
\hline Rama & 0.130106 & - & 211.49 & 213.49 & 0.324 & 0.000 \\
\hline Lindley & 0.0702 & - & 238.38 & 240.38 & 0.401 & 0.000 \\
\hline Exponential & 0.4079 & - & 261.73 & 263.73 & 0.447 & 0.000 \\
\hline
\end{tabular}

Table 2: MLE's, - 2ln L, AIC, K-S Statistics and p-values of the fitted distributions

It is quite obvious from table 2 that WRD is competing well with two parameter lifetime distributions and gives a quite satisfactory fit over the considered distributions. This means that, like other two-parameter lifetime distributions, WRD is also an important two-parameter lifetime distribution for modeling real lifetime data.

\section{Concluding Remarks}

A two-parameter weighted Rama distribution (WRD) which includes one parameter Rama distribution proposed by Shanker [1] has been suggested for modelling lifetime data from engineering. Its statistical properties including shapes of the probability density function for varying values of parameters, coefficients of variation, skewness, kurtosis, and index of dispersion have been studied. Its reliability measures including hazard rate function, mean residual life function, and the stochastic ordering have been discussed. The method of maximum likelihood has been discussed for estimating its parameters. The goodness of fit of the proposed distribution has been explained with a real lifetime data from engineering and the fit has been found quite satisfactory over one parameter exponential, Lindley and Rama distributions and two- parameter gamma, Gompertz, generalized exponential, lognormal, weighted Sujatha and weighted Lindley distributions.

\section{Acknowledgments}

Authors are grateful to the Editor-In-Chief of the Journal and the anonymous reviewers for their constructive comments which improved the quality and the presentation of the paper.

\section{References}

[1] R. Shanker, Rama distribution and Its Application, Int. J. Stat. Appl., 7(1) (2017), 26-35.

[2] R. A. Fisher, The effects of methods of ascertainment upon the estimation of frequencies, Ann. Eugenics, 6 (1934), $13-25$.

[3] C. R. Rao, On discrete distributions arising out of methods of ascertainment In: Patil, G.P.(eds) Classical and Contagious Discrete Distributions, Statistical Publishing Society, Calcutta, 1965, pp.320 - 332.

[4] G. P. Patil, C. R. Rao, The Weighted distributions: A survey and their applications, In applications of Statistics (Ed P.R. Krishnaiah0, North Holland Publications Co., Amsterdam, 1977, pp.383-405.

[5] G. P. Patil, C. R. Rao, Weighted distributions and size-biased sampling with applications to wild-life populations and human families, Biometrics, $\mathbf{3 4}$ (1978), $179-189$.

[6] R. D. Gupta, D. Kundu, Generalized exponential distribution, Aust. New Zealand J. Stat., 41(2) (1999), 173-188.

[7] M. E. Ghitany, F. Alqallaf, D. K. Al-Mutairi, H. A. Husain, A two-parameter weighted Lindley distribution and its applications to survival data, Mat. Comput. Simulation, 81 (2011), 1190-1201

[8] R. Shanker, K. K. Shukla, A Two-Parameter Weighted Sujatha Distribution and its application, Int. J. Appl. Math., 57(3) (2018), 106-121.

[9] M. G. Bader, A. M. Priest, Statistical Aspects of Fiber and Bundle Strength in Hybrid Composites, In; T. Hayashi, K. Kawata, S. Umekawa, (Eds), Progress in Science in Engineering Composites, ICCM-IV, Tokyo, 1982, pp. 1129 - 1136.

[10] M. Shaked, J. G. Shanthikumar, Stochastic Orders and Their Applications, Academic Press, New York, 1994.

[11] M. E. Ghitany, B. Atieh, S. Nadarajah, Lindley distribution and its Application, Mat. Comput. Simulation, 78 (2008), $493-506$ 
[12] D. V. Lindley, Fiducial distributions and Bayes' theorem, J. Royal Stat. Soc., Series B, 20 (1958), 102- 107.

[13] R. Shanker, F. Hagos, S. Sujatha, On modeling of Lifetimes data using exponential and Lindley distributions, Biometrics Biostat. Int. J., 2(5) (2015), 1-9. 